\title{
End-to-End Service Monitoring for Zero-Touch Networks
}

\author{
Nathan F. Saraiva de Sousa ${ }^{1, *}$, Danny Lachos Perez ${ }^{1}$, \\ Christian Esteve Rothenberg ${ }^{1}$ and Pedro Henrique Gomes ${ }^{2}$ \\ ${ }^{1}$ University of Campinas (UNICAMP), São Paulo, Brazil \\ ${ }^{2}$ Ericsson Research, São Paulo, Brazil \\ E-mail:nsaraiva@dca.fee.unicamp.br; dlachosp@dca.fee.unicamp.br; \\ chesteve@dca.fee.unicamp.br; pedro.henrique.gomes@ericsson.com \\ ${ }^{*}$ Corresponding Author
}

Received 01 November 2020; Accepted 02 March 2021; Publication 26 May 2021

\begin{abstract}
Autonomous management capability is the main pillar for paving Zerotouch Networks and efficiently deliver and operate use cases under the light of $5 \mathrm{G}$ requirements. To this end, Closed Control Loop (CCL), IntentBased Networking (IBN), and Machine Learning (ML) are regarded as enablers to automatically executed all operational processes, ideally without human intervention. In this context, the ETSI Zero-touch network and Service Management (ZSM) framework specifies an end-to-end network and service management reference architecture for managing the full lifecycle of services. However, the whole process of service monitoring is not yet well-consolidated in ETSI ZSM. In this work, we propose the Monitoring Model Generator (MMG) component to automatically construct templates for service monitoring. MMG implements a novel methodology where service deployment models and standard information models are used as inputs to generate a high-level monitoring template, called Service Monitoring Model (SMM) and built upon an ontology-based schema based on the Resource Description Framework (RDF) vocabulary. We present a proof of concept implementation along with an experimental functional validation of the
\end{abstract}

Journal of ICT Standardization, Vol. 9_2, 91-112. River Publishers

doi: 10.13052/jicts2245-800X.923

This is an Open Access publication. (C) 2021 the Author(s). All rights reserved. 
92 N. Saraiva et al.

MMG and using RDF data in turtle syntax and format. The resulting monitoring models are then used to define actual monitoring KPIs and construct management policies in a control loop architecture.

Keywords: Network management, Zero-Touch Network, IBN, Monitoring.

\section{List of Notations and Abbreviations}

3GPP Third Generation Partnership

5G Fifth Generation

AI Artificial Intelligence

CCL Closed Control Loop

DMG Deployment Model Generator

DSL Domain-Specific Language

E2E End-to-End

ETSI European Telecommunications Standards Institute

GST Generic Network Slice Template

IBN Intent-Based Networking

IM Information Model

KPI Key Performance Indicator

MD Management Domain

ML Machine Learning

MMG Monitoring Model Generator

NFV Network Function Virtualization

NMRGNetwork Management Research Group

NS Network Service

NSD Network Service Descriptor

OAM Operation, Administration, and Maintenance

PoC Proof of Concept

RDF Resource Description Framework

SDM Service Deployment Model

SDN Software Defined Networking

SDO Standards Developing Organization

SLA Service Level Agreement

SLO Service Level Objective

SLS Service Level Specification 
SMM Service Monitoring Model

VL Virtual Link

VNF Virtualised Network Function

ZSM Zero-touch network and Service Management

\section{Introduction}

Relevant technologies such as Software Defined Networking (SDN), Network Function Virtualization (NFV), and cloud computing, introduce new means for efficient, programmable, flexible utilization of their infrastructures through an entirely software-centric service paradigm [8]. These technologies together with network slicing are considered pillars for paving the fifth generation of mobile communication networks (5G) [5].

However, the benefits of flexibility, programmability, and efficiency provided by these enablers raise the complexity in operating and managing $5 \mathrm{G}$ networks. Traditional network management methods based on static configurations and manual processes are not adequate in highly dynamic environments (e.g. 5G slices). Therefore, new approaches are required towards improving how network operators use and maintain their networks providing features such as $(i)$ closed-loop automation in operating and managing the network and services (e.g., self-configuration, self-healing, self-optimization, and self-protecting), and (ii) service assurance increasing their added value.

Tasks related to Operation, Administration, and Maintenance (OAM) without human intervention can be implemented through autonomous Closed Control Loops (CCLs). CCLs along with Artificial Intelligence (AI) and Intent-based networking (IBN) approaches are being considered as enabling concepts of zero-touch networks. In this context, the Zero-touch network and Service Management (ZSM) initiative under development by ETSI targets End-to-End (E2E) service and network management automation in multidomain environments. ETSI ZSM defines a reference architecture to provide zero-touch automated network and service management in next-generation networks, such as 5G networks and beyond [12]. In order to enable a fully automated network and service management, the ZSM architecture is based on a set of principles, such as closed-loop management automation and intent-based interfaces. Figure 1 depicts the ZSM Management Domain (MD) presenting the main data services and their interactions.

Due to the $5 \mathrm{G}$ service types and their exigent requirements, the performance of an E2E service is of huge importance, and constant performance monitoring becomes rather crucial to assure the desired requirements. The 


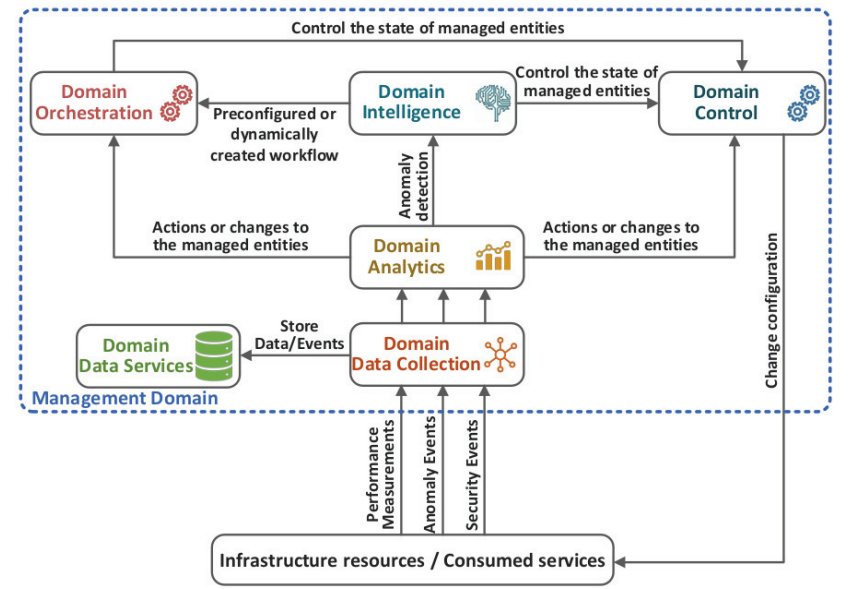

Figure 1 Architectural Overview of ZSM. Scope of management outlined by a technological or organizational boundary [6].

translation from intent-based services requirements into KPIs and configurations for automated monitoring is not yet a well-consolidated approach inside ZSM. Besides, much of the standardization initiatives such as ETSI NFV/MEC [10, 9], 3GPP [1], GSMA [4], TM Forum [15], and IETF NMRG [7, 17] are mainly focused on the deployment process of network services, leaving aside the whole process of monitoring, mainly the E2E KPIs monitoring. Therefore, it is essential to have a common method that providers and operators can use in order to monitor the performance of any end-to-end service.

In this work, we propose a novel methodology to generate a Service Monitoring Model (SMM) for a requested intent-based E2E service. The SMM is holistic enough to enable a higher-level abstraction of the managed environment, decoupling the service monitoring from the current management tools, especially in E2E scenarios. SMM defines the KPIs and attributes with values and/or ranges for monitoring a specific service based on customer requirements and proposed monitoring ontology. The proposed method extends the ZSM architecture to include service monitoring as a parallel process to service deployment and integrated into the closed loop-based E2E management.

The main contributions of this paper are as follows:

- Comprehensive overview of standardization efforts for modeling features of E2E service deployment and monitoring. 
- Proposal of E2E service monitoring methodology based on a domainspecific ontology.

- Functional validation of the proposed method in a proof of concept implementation aligned with ETSI ZSM.

The rest of the paper is organized as follows. Section 2 presents standardization activities describing features in E2E service deployment and monitoring. Section 3 describes in detail the Monitoring Model Generator (MMG) component, along with the generation of an abstract level template known as a Service Monitoring Model - SMM (Section 4). We point out the implementation of a Proof of Concept (PoC) prototype in Section 5 before concluding the paper in Section 6.

\section{Related Work}

CCLs require constant monitoring and Service Level Agreements (SLAs) verification. How to provide such continuous monitoring and service assurance methods is an open problem that is evaluated by different Standards Developing Organizations (SDOs). However, much of the efforts are focused on the deployment process of E2E services, leaving aside the whole process of monitoring the services and E2E chains of Virtual Network Functions (VNFs). In this section, we review current initiatives on standardization bodies for modeling E2E service deployment and monitoring. Table 1 summarizes the main standardization efforts for expressing features of service deployment and monitoring.

\subsection{ETSI ZSM}

The ETSI Zero-Touch network and Service Management Industry Specification Group (ZSM ISG) has designed a ZSM framework supporting full automated network and service management in multi-domain environments [6]. The ETSI ZSM framework includes all the operational processes, such as design/planning, delivery, deployment, provisioning, monitoring, and optimization, which are in turn automatically executed with $0 \%$ human intervention.

The ETSI ZSM reference architecture [12] is based on a model-driven approach to perform the management of services/resources using information models. The models capture attributes and supported operations of resources and services, and they are independently defined from the implementation to increase portability and reusability. Currently, ETSI ZSM is exploring 
Table 1 Summary of standardization efforts around end-to-end multi-domain service deployment and monitoring

\begin{tabular}{|c|c|c|c|c|c|c|c|c|}
\hline \multirow[b]{2}{*}{ SDO } & \multirow[b]{2}{*}{ Purpose } & \multirow[b]{2}{*}{ Scope } & \multicolumn{4}{|c|}{ Metrics } & \multicolumn{2}{|c|}{ Function } \\
\hline & & & Edge & VNF & VIM & E2E & Deploy & Monitor \\
\hline $\begin{array}{l}\text { ETSI } \\
\text { ZSM }\end{array}$ & $\begin{array}{l}\text { A model-driven approach } \\
\text { to perform service and } \\
\text { resource management }\end{array}$ & $\begin{array}{l}\text { SDN, } \\
\text { NFV, } \\
\text { Network } \\
\text { Slicing }\end{array}$ & $\checkmark$ & $\checkmark$ & $\checkmark$ & $\checkmark$ & 0 & 0 \\
\hline $\begin{array}{l}\text { ETSI } \\
\text { NFV- } \\
\text { IFA }\end{array}$ & $\begin{array}{l}\text { Deployment template } \\
\text { which consists of } \\
\text { parameters used by } \\
\text { the NFVO during lifecycle } \\
\text { of an NS }\end{array}$ & NFV & & $\checkmark$ & $\checkmark$ & & 0 & D \\
\hline $\begin{array}{l}\text { GSMA } \\
\& \\
3 \mathrm{GPP}\end{array}$ & $\begin{array}{l}\text { 5G network slice tem- } \\
\text { plates with attributes that } \\
\text { describe the service char- } \\
\text { acteristics }\end{array}$ & $\begin{array}{l}\text { Network } \\
\text { Slicing }\end{array}$ & & & & $\checkmark$ & - & - \\
\hline $\begin{array}{l}\text { ETSI } \\
\text { MEC }\end{array}$ & $\begin{array}{l}\text { Application templates for } \\
\text { MEC environments that } \\
\text { specify resources and ser- } \\
\text { vice lifecycle aspects. }\end{array}$ & MEC & $\checkmark$ & $\checkmark$ & $\checkmark$ & & 0 & 0 \\
\hline $\begin{array}{l}\mathrm{TM} \\
\text { Forum }\end{array}$ & $\begin{array}{l}\text { Service requests and } \\
\text { lifecycle management } \\
\text { through standard APIs }\end{array}$ & $\begin{array}{l}\text { SDN, } \\
\text { NFV }\end{array}$ & & $\checkmark$ & $\checkmark$ & $\checkmark$ & - & D \\
\hline $\begin{array}{l}\text { IRTF } \\
\text { NMRG }\end{array}$ & $\begin{array}{l}\text { Express service needs } \\
\text { using } \\
\text { mechanisms }\end{array}$ & SDN & & & & $\checkmark$ & - & D \\
\hline
\end{tabular}

different SDOs (e.g., ETSI ISG NFV, TM Forum, 3GPP) to identify use cases, architectural components, interfaces, information models, and templates that may be relevant to the work in ZSM [14]. In the particular case of ETSI ISG NFV, interfaces and information models have been identified to be leveraged in ZSM to management the E2E services (lifecycle, fault, performance, and policy).

\subsection{ETSI NFV-IFA}

The Interfaces and Architecture (IFA) Working Group (WG) in ETSI NFV describes the ETSI NFV architecture, information models, functional requirements, and normative Network Service Descriptors (NSDs) [10]. In particular, the NSD is used by an NFV orchestrator to deploy a network service (NS) instance. The NSD is a deployment template describing an NS as a composition of VNFs and virtual links (VLs). Both VNFs and VLs contain 
a set of attributes to assist the resource instantiation for functions and links, respectively.

The VNFs and VLs have their corresponding descriptors to specify a list of constraints (e.g., QoS properties) and monitoring (e.g., VNF scaling) parameters. Monitoring parameters can also be specified at the NS level, however, E2E monitoring metrics are out of the specification scope.

\subsection{GSMA \& 3GPP}

Evolving network scenarios (e.g., 5G) bring new challenges in terms of performance and capabilities for traditional operator networks [16]. One of those challenges is the ability to partition one common network infrastructure into multiple independent virtual E2E networks; an approach referred to as network slicing. Each slice is then used as a dedicated network by vertical customers to allocate resources in all the distinct network segments (i.e., core, transport, and access networks).

In the 3GPP architecture, management systems will use a service profile to request an E2E network slice. The service profile is derived from a Generic Network Slice Template (GST), specified by the GSM Association (GSMA). The GST's general structure includes optional, conditional, and mandatory attributes to implement slices, including the expected SLOs and scalability characteristics.

In the case of monitoring and supervision aspects, the 3GPP architecture supports a complete set of generic Management Services (MnS) to support different requirements on network performance, network fault supervision, among others [13]. Management systems, for instance, can use Performance Monitoring (PM) services to optimize network performance or Fault Management (FM) services to prevent/predict network failures.

\subsection{ETSI Multi-access Edge Computing (MEC)}

The Application Descriptor (AppD) [9], specified by the ETSI MEC, is used to describe the application rules and requirements of a MEC application, including the application lifecycle management. Specifically, the AppD contains a description of minimum computation, storage, and network resources, along with other aspects such as a description of traffic and DNS rules.

The deployment of MEC in an NFV environment is also possible [11]. Therefore, NSDs and AppDs can co-exist to allow mobile edge components and applications to be instantiated in a virtualized infrastructure. 


\subsection{TeleManagement Forum (TM Forum)}

In order to facilitate the interaction between network providers and applications, the TM Forum has been working to develop a set of open APIs to request services with the required management functionality [15].

The Service Catalog Management API (TMF633), for example, allows the management of the entire lifecycle of the catalog elements which are available through services that an operator offers to the customers. The Service Ordering API (TMF641) specifies a model definition to request a service order including operations such as creating, updating, retrieving, and filtering. The Service Inventory API (TMF638) provides a standardized mechanism to query and manipulate a customer's service instances. Finally, the Service Activation and Configuration API (TMF640) enables the creation, modification, and termination of service instances. Such lifecycle management actions include the collection of monitoring data through the definition of threshold/periodic-based rules.

\subsection{IRTF Network Management Research Group (NMRG)}

The IRTF NMRG is defining concepts for an Intent lifecycle with two functional planes: fulfillment and Assurance [7]. The Intent Fulfillment plane provides the necessary functions and interfaces that take an intent from its origination by a user to its execution in the network. The Intent Assurance plane validates that the executed service is indeed adhering to the expectations specified in the intent. It also provides functions interfaces, including monitoring functions (e.g., performance and events outliers) to assess whether the observed behavior complies with the desired intent.

Currently, there is no common agreement on the model/interface of an intent, especially due to the diversity of intent stakeholders, targets, network scopes, and infrastructure. Based on such parameters, the NMRG is working to clarify the definition of an intent, including an intent classification [17].

\section{Monitoring Model Generator (MMG)}

The Monitoring Model Generator (MMG) component proactively constructs templates for service monitoring, called service monitoring model (SMM). The SMM is generated from the Service Deployment Model (SDM), together with the information model (IM) obtained from main SDOs such as 3GPP and ETSI. In addition, specific measurable characteristics of the service could be taken from an SLA as well. Instead of individually monitoring 
all metrics and elements that compose a service (e.g., VNFs, VLs), the proposed method automatically selects the metrics to be evaluated based on the service intent and KPIs that better represent the behavior of the service to be monitored. Such metrics should be the most representative in terms of end-to-end network service quality.

The SMM can be used by the control plane, which represents one or more operational and management entities using the CCL functionality, to repeatedly verify the effectiveness of the previously deployed service. This methodology allows decoupling the monitoring components of the E2E service from its deployment. This decoupling brings advantages for the evolution of the management systems, mainly for new service assurance based on closed loops.

The proposed approach brings three main advantages when compared to the state-of-the-art management systems:

- Modularity. The two different models, i.e., deployment and monitoring models, are decoupled. This reduces the complexity of the overall management and allows them to work and evolve independently. This is essential for a closed-loop automation where pieces of the closed loop (monitoring, analytics, decision, orchestration, etc.) come from different vendors and can be connected in different ways;

- Simplicity. The E2E network service design can be simplified because vendors that perform the service deployment do not need to worry about the details of the service monitoring;

- Portability. The monitoring models are agnostic to the traffic and can be reused by different applications. They are use case-independent, which facilitates their portability and reusability. The same techniques and patterns for creating monitoring models can be applied to different network domains, e.g., access, core, cloud, etc.

\subsection{System Architecture}

The work objective is mainly focused on the MMG component that provides the steps of (i) receiving an SDM from an intent-based service requirement, (ii) a high-level IM, and (iii) service requirements in the form of an SLA. The MMG component, or simply MMG, processes those inputs for automatically creating an SMM. Figure 2 illustrates the actors involved in an SMM generation from the beginning to the end and contextualizes the overall scenario. The parts in gray are out of the scope of the present paper, i.e., Deployment Model Generator (DMG). The rest (in colorful) represent the components that 


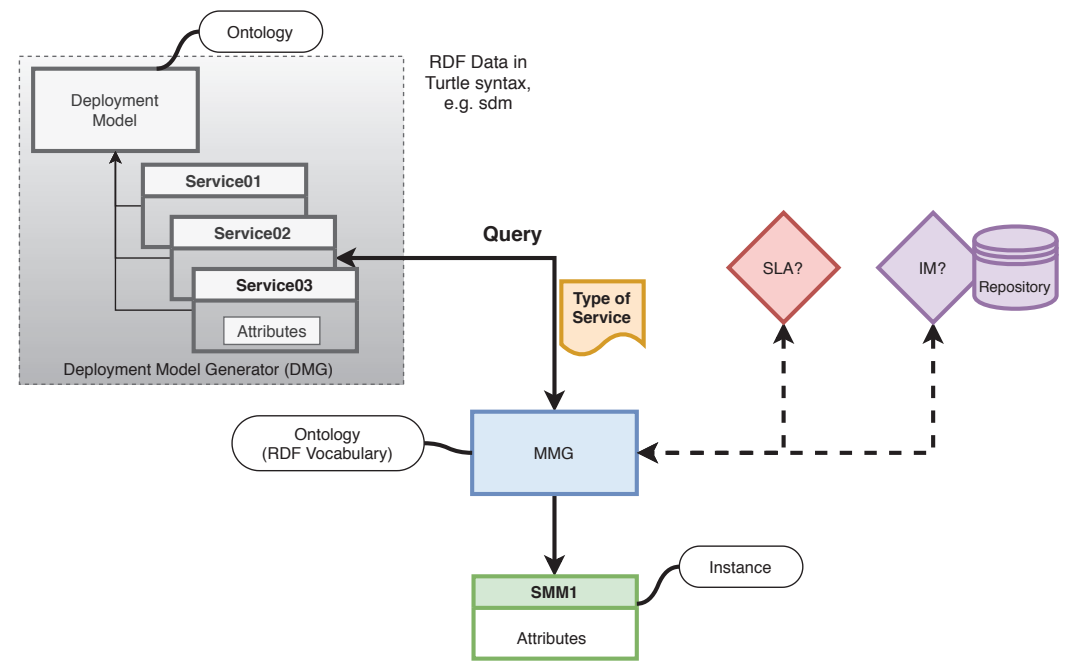

Figure 2 System Architecture.

are the focus of this work and their interactions (e.g., IM repository, SLA, MMG).

The MMG uses the information contained in the SDM to, together with SLA or IM or both and modeled through an RDF vocabulary, compose an individual instance of the SMM. Once SMM is generated, the control plane uses it to manage the lifecycle of the service.

The Information Model (IM) Repository contains different information model templates. Those templates might refer to several industry standards, such as 3GPP SA5, TM Forum, ETSI NFV MANO, OASIS TOSCA. Information model templates might also refer to models specified by projects aligned with open source projects (e.g., ONAP, OSM) and main vendors. MMG can use one or more information models in the building of the monitoring template.

Formal or informal contracts are expressed in the form of SLAs. An SLA is an electronic document that specifies what the customer should expect from the service provider through functional or non-functional properties and the consequences in case of violation. There is no standard model to create an SLA, but it briefly consists of measurable metrics, also defined as Service Level Objective (SLO). A set of SLOs constitutes a Service Level Specification (SLS). An SLO refers to a performance metric and specifies a value or value ranges and unit along with a condition, for instance, availability $\geq 99 \%$, end-to-end delay between 50-100 ms. 


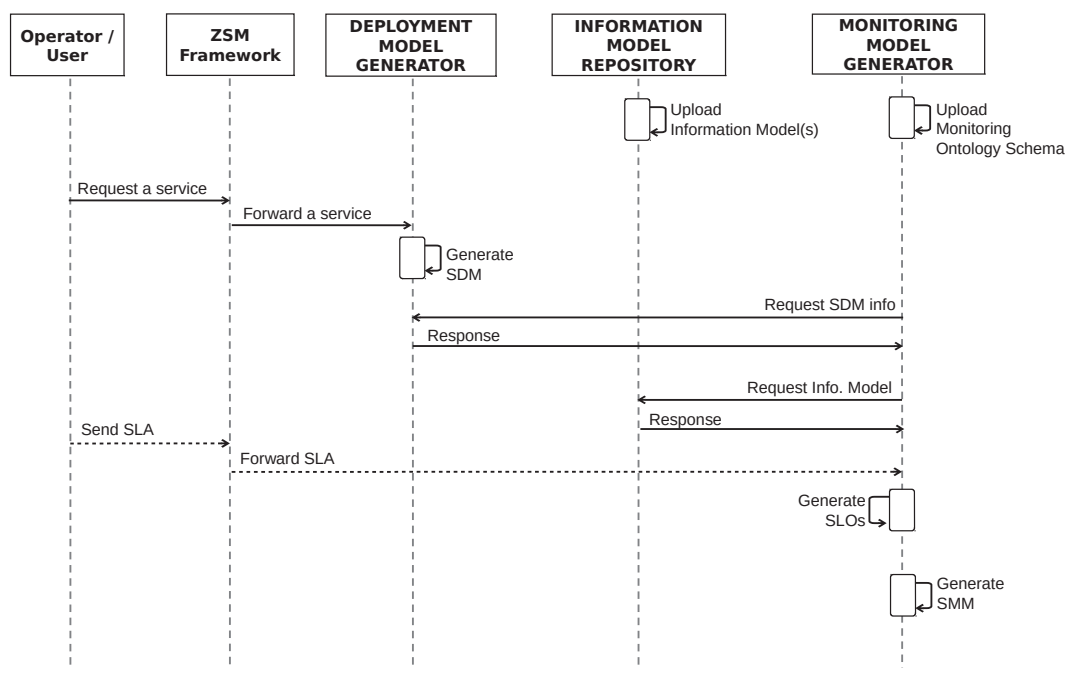

Figure 3 Sequence diagram to generate the an instance of service monitoring model.

\subsection{High-level Monitoring Template}

Figure 3 provides overall steps for the generation of an instance of SMM. This work is mostly focused on the step in which the SMM is the output generated by the MMG component running the proposed method. Initially, the information models from different standardization organizations, research projects, and vendors are inserted into the IM repository as well as monitoring ontology is loaded into MMG.

A user/operator requests an intent-based service to the ZSM framework, and it, in turn, forwards the request to the deployment model generator (DMG). It generates an instance of SDM from information contained in the request modeled through an ontology-based schema. The SDM must contain specific details of E2E service (e.g., streaming audio and video, voice), constraints (e.g., start time, date), and connection points.

The MMG queries SDM instance to retrieve information about the requested service, such as the type of service and duration. So, the Information Model (IM) repository should be queried to select the relevant metrics that impact the service quality. Eventually, SLAs could be provided by the user/operator as an input to the MMG to specify the SLOs such as availability, throughput, frequency, response time, or quality of service. SLOs defined by an SLA may not always reflect the key monitoring metrics of service so that the system can complement them with SLOs defined by IM. However, 
SLOs defined by an SLA has greater priority over SLOs defined by IM. The MMG will generate an instance of SMM from an ontology-based schema, more specifically an RDF vocabulary. This ontology-based schema allows capturing several metrics and conditions (including time constraints) obtained from IM, SLA, or both.

\section{Service Monitoring Model}

An ontology defines a common vocabulary for sharing information in a specific domain including definitions of basic concepts and relations among them. It is composed of a formal description of concepts (classes) in a domain, properties of each concept (properties), and restrictions on properties (role restrictions). An ontology is implemented through a domain-specific language (DSL) such as Resource Description Framework (RDF).

The MMG component uses a semantic and an approach based on an RDF vocabulary to generate an abstract level template (i.e., an SMM) focused on monitoring of service, hence on the management of the service lifecycle. The proposed RDF vocabulary has a set of terms to describe the service monitoring domain, such as classes (and subclasses), properties, and relationships. In practice to build an RDF vocabulary (or ontology), firstly, we follow the steps: (i) define classes, i.e., specify the class name, comments, label (e.g., class Metric in Figure 4), (ii) arrange the classes hierarchically in a taxonomy (i.e., superclass and subclass as shown in Figure 4), and (iii) specify the properties and define values and ranges for them. We use the terms ontology and RDF vocabulary as synonyms in this paper.

Figure 4 represents a snapshot of a piece of the developed monitoring ontology. Basically, we arrange the ontology in 4 main classes (superclasses): (i) Metric, E2E metrics (or KPIs) in different contexts such as application, cloud and network; (ii) Service, E2E service including 5G services, e.g., video, health, automotive, etc, (iii) Slo, service level objective, and (iv) Unit, unit of measurements such as data transmission unit and energy unit. For the classes Service and Unit, we reuse and extend an existing ontology named Toucan Ontology (ToCo). ${ }^{1}$

Figure 5 shows classes, subclasses, and relations among them in the monitoring domain. The MMG defines individual instances of these classes (as an object of a class in the Object-Oriented programming) filling in specific properties value information and additional role restrictions. Each instance is

${ }^{1}$ https://qianruzhou333.github.io/toco_ontology/ 


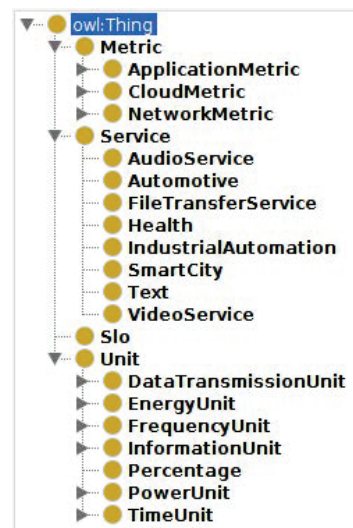

Figure 4 Taxonomic (subclass-superclass) hierarchy in the service monitoring domain.

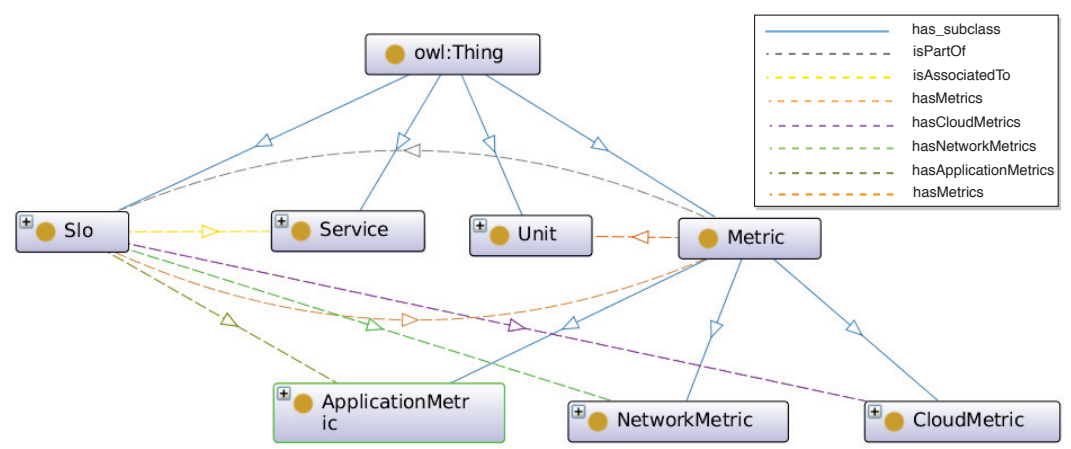

Figure 5 Relationships between classes and subclasses in the service monitoring domain.

related to a particular E2E service in execution. It is worth mentioning that the proposed ontology expresses a non-exhaustive set of classes and properties that can be extended when necessary.

\section{Prototype Implementation and Experimental Validation}

Targeting functional experimental validation, we developed a Proof of Concept $(\mathrm{PoC})$ implementation of the proposed architecture and monitoring components. As shown in Figure 6, we extend the ZSM architecture by adding a layer before service deployment, i.e., at design time. The proposed layer encompasses the MMG component and IM repository and is responsible for creating an instance of the SMM. The MMG output (i.e., SMM) is then 


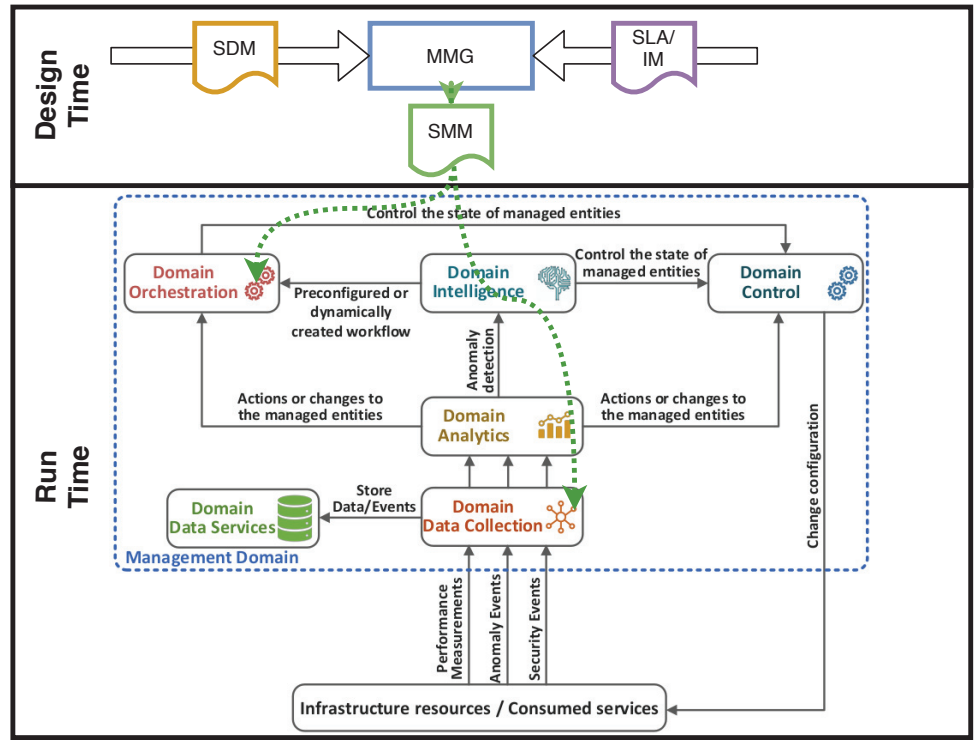

Figure 6 Mapping between PoC components and ZSM architecture.

used as input for both Domain Orchestration and Domain Data Collection components. Domain Orchestration uses the SMM to build the service policy condition whilst Data Collection processes the SMM to define the metrics to be monitored.

\subsection{PoC Details}

Although our focus is on the methodology to build the SSM, it is required to have an instance of SDM as input for MMG. For that, we develop a simple ontology in the domain of E2E service deployment. Figure 7 shows the main classes, properties, and relationships of the PoC ontology comprised of three superclasses: Customer, Provider, and Service. From these classes, individual instances are filled in specific attributes values. An example is represented in the yellow box of Figure 7. Each instance of the class Service has attributes such as an identifier, status, type, start time, and end time. MMG uses these attributes to create an instance of SMM.

An IM example based on 3GPP Information Model is provided as an illustrative and not restrictive choice for the PoC. More specifically, the documents used as reference are 3GPP TS 23.107 [2] and 3GPP TS 22.105 [3]. The first one specifies the list of metrics applicable in the 3GPP system, while 


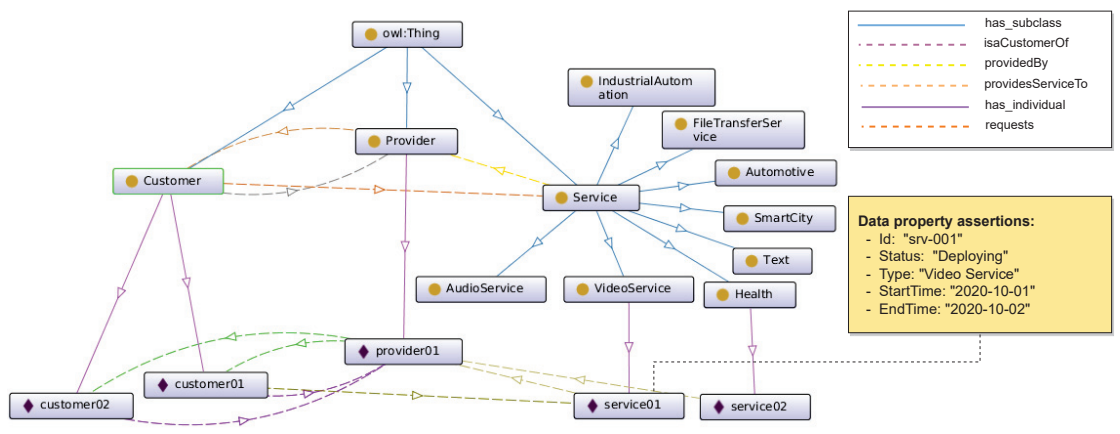

Figure 7 Sample of E2E service deployment model.

Table 2 E2E quality metrics for video service from 3GPP

\begin{tabular}{lccc}
\hline Metric & Condition & Value (or Range) & Unit \\
\hline Download throughput & - & $20-284$ & $\mathrm{kbps}$ \\
\hline Start-up delay & $<$ & 10 & $\mathrm{sec}$ \\
\hline Jitter & $<$ & 2 & $\mathrm{sec}$ \\
\hline Packet loss & $<$ & 2 & $\%$ \\
\hline
\end{tabular}

the second one describes how and what kind of services the user has access to. According these documents, the quality of service is defined with a set of metrics (attributes). However, the number of these metrics should be kept low (raising the number of metrics, raises service management complexity). Table 2 presents the video service quality metrics and their values (or range of values) according to the document [2]. These metrics and values are stored in a database to be queried by the MMG.

\subsection{Example Scenario}

Firstly, a customer requests an intent-based service to a provider01 through ZSM framework, "I want to transmit real-time video using the mobile network from point A to B from 2020-10-01 until 2020-10-02." The service request is processed and it is generated an instance of SDM in RDF format. For generating that instance, we use the Protégé ${ }^{2}$ tool. MMG queries the SDM (RDF data) to retrieve information about the requested service including class (i.e., type of service), id, status, start time and start time. For query, we use a SPARQL request/response.

\footnotetext{
${ }^{2}$ https://protege.stanford.edu/
} 
- SPARQL Request

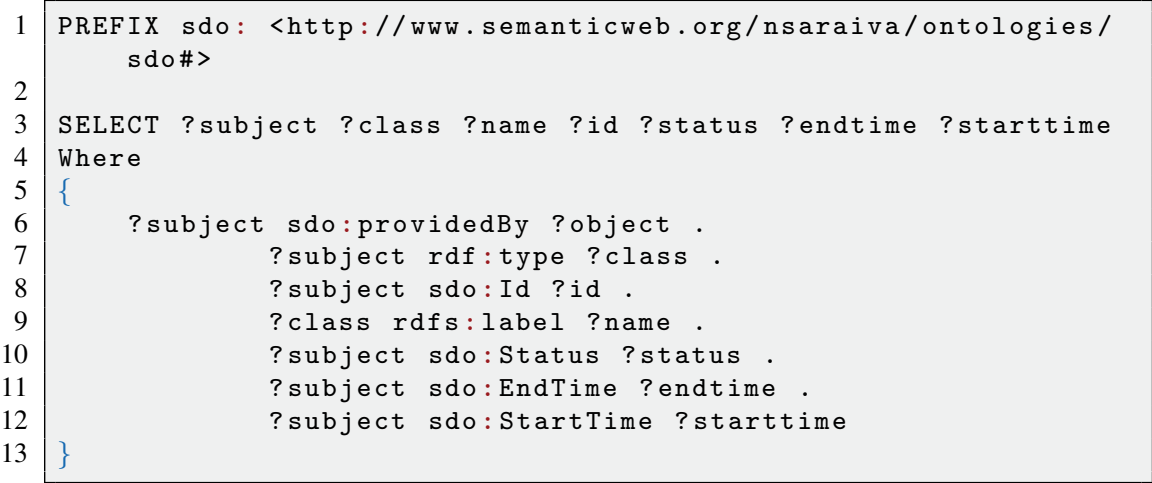

\section{- SPARQL Response}

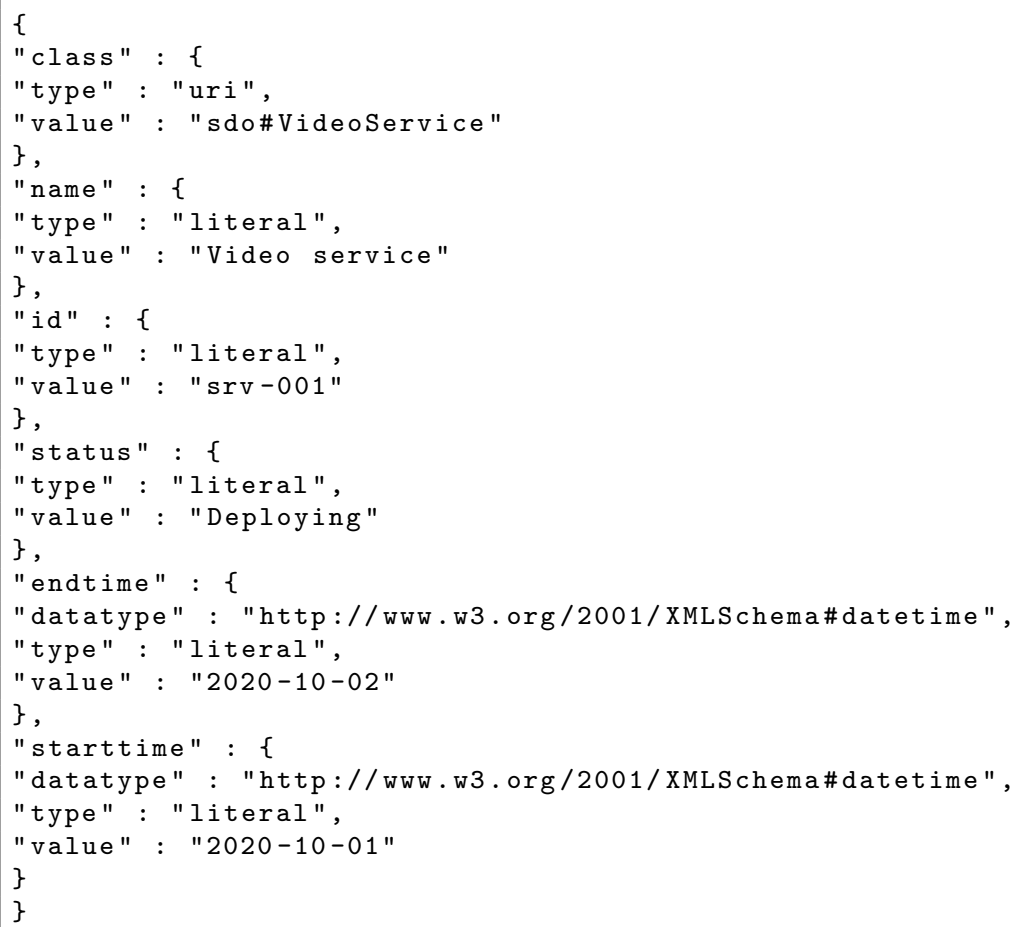

The customer does not send the service SLA, so an IM is required to derive the metrics related to the target service. For our example, we use the 3GPP IM shown in Table 2. Based on that IM, on information collected from 


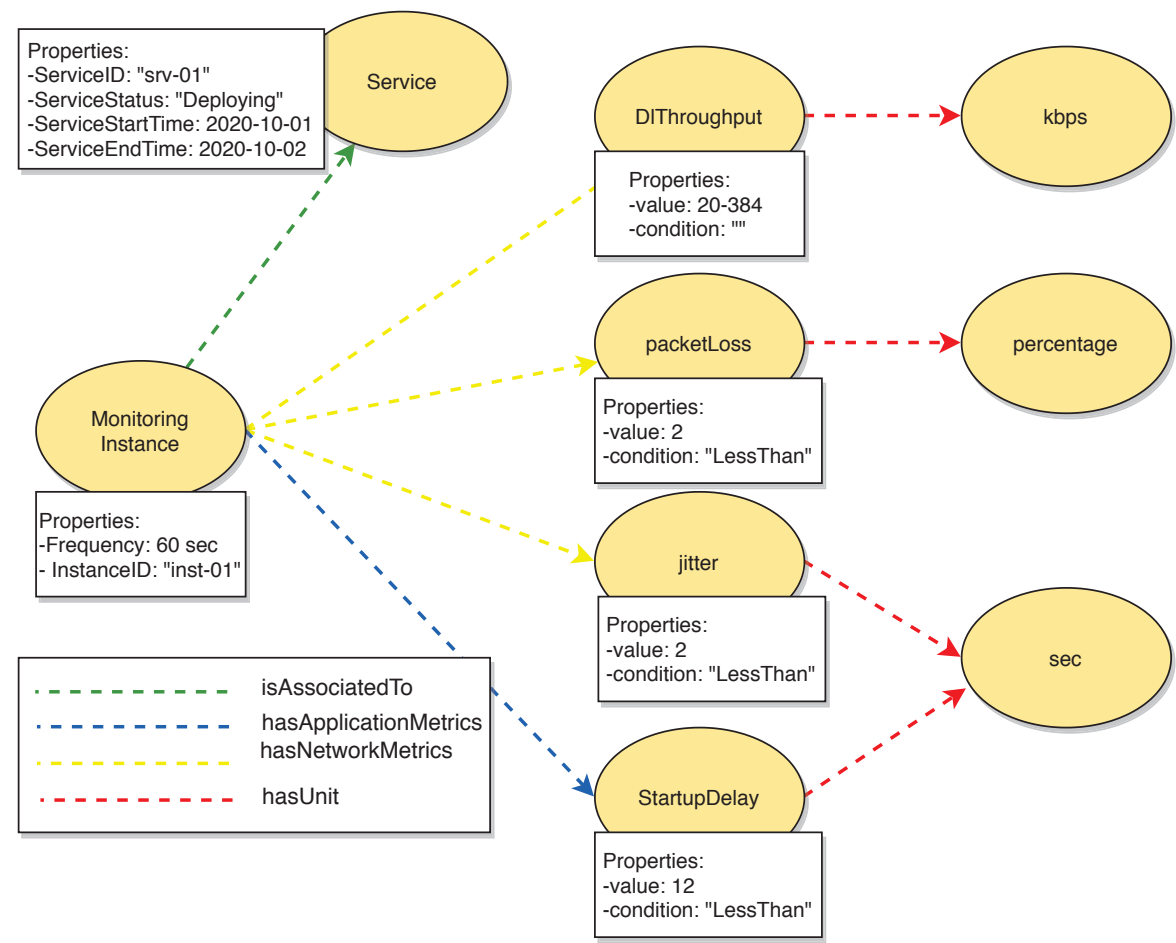

Figure 8 Instance of Monitoring model generated automatically by the MMG component using 3GPP IM.

SDM, and on template presented in Figure 4, MMG generates an instance of service monitoring model illustrated in Figure 8. From that model, the control plane may manage the lifecycle of the requested service properly.

\section{Conclusions and Future Work}

Emerging networking scenarios such as those envisioned for $5 \mathrm{G}$ network slicing demand constant service performance monitoring to assure specific requirements. In this work, we designed and implemented a methodology for E2E service monitoring inspired by the ETSI ZSM architecture. Starting with an intent-based service request, it a monitoring template is automatically generated using an ontology-based schema without including details of the network infrastructure, which facilitates portability and reusability. 
The functional validation experiments bring essential guidelines for creating a knowledge base composed of individual instances of SMM to correlate services with performance metrics and eventually inference new correlations (e.g., SLA violation with potential root causes) through knowledge reasoning. Other future activities include enhancing the service monitoring ontology and apply the monitoring model on a real use case using a larger scale control loop platform.

\section{Acknowledgment}

This research was supported by the Innovation Center, Ericsson S.A., Brazil, grant UNI.67. The views expressed are solely those of the authors and do not necessarily represent Ericsson's official standpoint.

\section{References}

[1] 3GPP. Management and orchestration; provisioning. Technical report, TS 28.351.

[2] 3GPP. Technical specification group services and system aspects: quality of service (QoS) concept and architecture. Technical report, TS 23.107.

[3] 3GPP. Technical specification group services and system aspects: Services and service capabilities. Technical report, TS 22.105.

[4] GSM Association. Generic network slice template. https://www.gsma .com/newsroom/wp-content/uploads//NG.116-v2.0.pdf, 2019.

[5] Alcardo Alex Barakabitze, Arslan Ahmad, Rashid Mijumbi, and Andrew Hines. 5g network slicing using sdn and nfv: A survey of taxonomy, architectures and future challenges. Computer Networks, 167:106984, 2020.

[6] Chafika Benzaid and Tarik Taleb. Ai-driven zero touch network and service management in $5 \mathrm{~g}$ and beyond: Challenges and research directions. IEEE Network, 34(2):186-194, 2020.

[7] Alexander Clemm, Laurent Ciavaglia, Lisandro Zambenedetti Granville, and Jeff Tantsura. Intent-Based Networking - Concepts and Definitions. Internet-Draft draft-irtf-nmrg-ibn-concepts-definitions-02, Internet Engineering Task Force, September 2020. Work in Progress. 
[8] Nathan F Saraiva de Sousa, Danny A Lachos Perez, Raphael V Rosa, Mateus AS Santos, and Christian Esteve Rothenberg. Network service orchestration: A survey. Computer Communications, 142:69-94, 2019.

[9] ETSI. Multi-access edge computing (MEC); framework and reference architecture. https://www.etsi.org/deliver/etsi_gs/MEC/001_099/003/0 2.01.01_60/gs_MEC003v020101p.pdf.

[10] ETSI. Network Functions Virtualisation (NFV); Management and Orchestration; Network Service Templates Specification . https://ww w.etsi.org/deliver/etsi_gs/NFV-IFA/001_099/014/02.01.01_60/gs_NFV -IFA014v020101p.pdf, 2016.

[11] ETSI. Mobile edge computing (MEC);deployment of mobile edge computing in an NFV environment. https://www.etsi.org/deliver/ets i_gr/MEC/001_099/017/01.01.01_60/gr_MEC017v010101p.pdf, 2018.

[12] ETSI. Zero-touch network and Service Management (ZSM); Reference Architecture. https://www.etsi.org/deliver/etsi_gs/ZSM/001_099/002/0 1.01.01_60/gs_ZSM002v010101p.pdf, 2019.

[13] ETSI. 5G; Management and orchestration; Architecture framework (3GPP TS 28.533 version 15.3.0 Release 15). https://www.etsi.org /deliver/etsi_ts/128500_128599/128533/15.03.00_60/ts_128533v150300 p.pdf, 2020.

[14] ETSI. Zero-touch network and Service Management (ZSM); Landscape. https://www.etsi.org/deliver/etsi_gr/ZSM/001_099/004/01. 01.01_60/gr_ZSM004v010101p.pdf, 2020.

[15] TM Forum. TM Forum Open APIs. https://projects.tmforum.org/wiki/ display/API/Open+API+Table.

[16] Kai Gao, Luis M. Contreras, and Sabine Randriamasy. Bi-directional network and application interaction: Application intents upon abstracted network information (invited paper). In Proceedings of the Workshop on Network Application Integration/CoDesign, NAI'20, page 43-50, New York, NY, USA, 2020. Association for Computing Machinery.

[17] Chen Li, Olga Havel, Will (Shucheng) LIU, Adriana Olariu, Pedro Martinez-Julia, Jéferson Campos Nobre, and Diego Lopez. Intent Classification. Internet-Draft draft-irtf-nmrg-ibn-intent-classification-00, Internet Engineering Task Force, July 2020. Work in Progress. 


\section{Biographies}

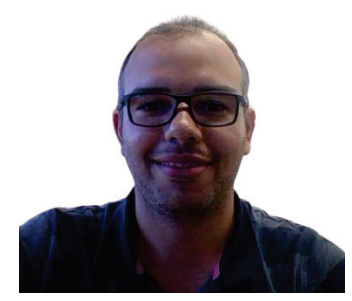

Nathan F. Saraiva de Sousa has a BSc and an MSc in Computer Science from Federal University of Piaui (UFPI), in 2005 and 2015, respectively. He is currently a Ph.D. candidate at the Department of Computer Engineering and Industrial Automation of the School of Electrical and Computer Engineering - UNICAMP. He works in a project funded by Ericsson Research Brazil. Since 2010 he is a Computer Analyst at the Federal Institute of Piaui (IFPI). His research interests span SDN, NFV, and multi-domain orchestration.

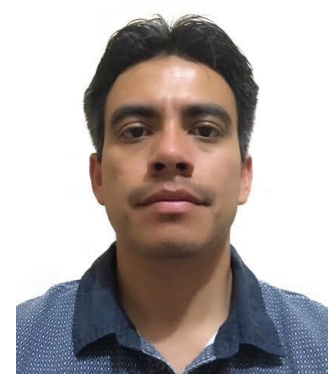

Danny Lachos Perez is a Ph.D. candidate in the Faculty of Electrical and Computer Engineering at University of Campinas (UNICAMP). He is involved as a software and Internet researcher in different academy-industry collaboration projects related to networked systems (e.g., 5G, SDN, NFV, data center networking, Internet routing). Danny has a particular interest in new and flexible network and application integration mechanisms in the context of multi-domain environments. He holds the Computer Engineering degree from the Pedro Ruiz Gallo University (UNPRG), Peru, and the M.Sc. degree in Computer Engineering from UNICAMP, 2016. 


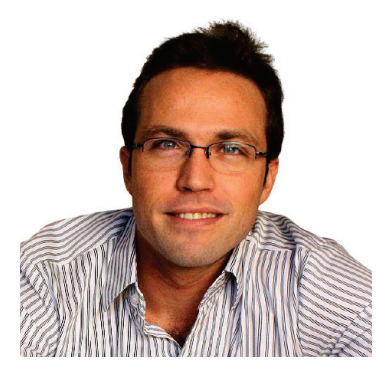

Christian Esteve Rothenberg is an Assistant Professor in the Faculty of Electrical \& Computer Engineering (FEEC) at University of Campinas (UNICAMP), Brazil, where he received his Ph.D. and currently leads the Information \& Networking Technologies Research \& Innovation Group (INTRIG). His research activities span all layers of distributed systems and network architectures and are often carried in collaboration with industry, resulting in multiple open source projects in SDN and NFV among other scientific results.

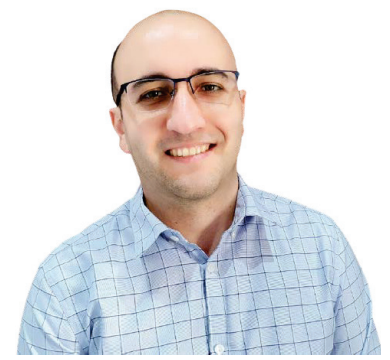

Pedro Henrique Gomes is a researcher at Ericsson Research, Brazil, engaged in orchestration and automation of $5 \mathrm{G}$ networks and services. He is a delegate in the ETSI Zero-Touch Network \& Service Management working group, contributing to the architecture definition especially with AI and ML concepts, and driving the specification of enablers for closed-loop automation in end-to-end network services. Received Ph.D. (2019) and M.Sc. (2015) in electrical engineering from the University of Southern California, Los Angeles, USA, also M.Sc. (2011) in computer science and B.Sc. (2007) in computer engineering from the University of Campinas, Brazil. 
